\title{
Testing the theory of grain growth and fragmentation by millimeter observations of protoplanetary disks
}

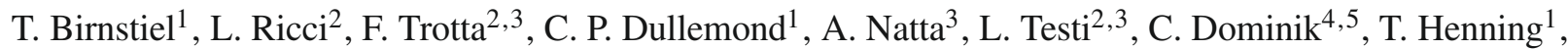 \\ C. W. Ormel ${ }^{1}$, and A. Zsom ${ }^{1}$ \\ 1 Max-Planck-Institut für Astronomie, Königstuhl 17, 69117 Heidelberg, Germany \\ e-mail: birnstiel@mpia.de \\ 2 European Southern Observatory, Karl-Schwarzschild-Strasse 2, 85748 Garching, Germany \\ 3 Osservatorio Astrofsico di Arcetri, INAF, Largo E. Fermi 5, 50125 Firenze, Italy \\ 4 Astronomical Institute "Anton Pannekoek", University of Amsterdam, PO Box 94249, 1090 GE Amsterdam, The Netherlands \\ 5 Afdeling Sterrenkunde, Radboud Universiteit Nijmegen, Postbus 9010, 6500 GL Nijmegen, The Netherlands
}

Received 20 April 2010 / Accepted 4 June 2010

\begin{abstract}
Context. Observations at sub-millimeter and mm wavelengths will in the near future be able to resolve the radial dependence of the $\mathrm{mm}$ spectral slope in circumstellar disks with a resolution of around a few AU at the distance of the closest star-forming regions.

Aims. We aim to constrain physical models of grain growth and fragmentation by a large sample of (sub-)mm observations of disks around pre-main sequence stars in the Taurus-Auriga and Ophiuchus star-forming regions.

Methods. State-of-the-art coagulation/fragmentation and disk-structure codes are coupled to produce steady-state grain size distributions and to predict the spectral slopes at (sub-)mm wavelengths.

Results. This work presents the first calculations predicting the mm spectral slope based on a physical model of grain growth. Our models can quite naturally reproduce the observed mm-slopes, but a simultaneous match to the observed range of flux levels can only be reached by a reduction of the dust mass by a factor of a few up to about 30 while keeping the gas mass of the disk the same. This dust reduction can either be caused by radial drift at a reduced rate or during an earlier evolutionary time (otherwise the predicted fluxes would become too low) or due to efficient conversion of dust into larger, unseen bodies.
\end{abstract}

Key words. accretion, accretion disks - circumstellar matter - stars: pre-main-sequence - protoplanetary disks planets and satellites: formation - submillimeter: stars

\section{Introduction}

Circumstellar disks play a fundamental role in the formation of stars as most of the stellar material is believed to be transported through the disk before being accreted onto the star (Lynden-Bell \& Pringle 1974). At the same time circumstellar disks are thought to be the birth places of planets. Understanding the physics of circumstellar disks is therefore the key to some of the most active fields of astrophysical research today.

However, observing these disks in order to learn about the physical processes taking place in their interior is a challenging task. Strom et al. (1989) and Beckwith et al. (1990) were the first to use observations at mm-wavelengths to confirm that many of the observed pre-main sequence (PMS) stars showed excess radiation above the spectrum of a T Tauri star. While these single dish observations provided valuable insight into dust masses (because mm observations probe not only the thin surface layers, but the bulk of the dust mass in the mid-plane), recent developments in the field of (sub-)mm interferometry allow one to constrain models of disk structure and evolution of protoplanetary disks by fitting parametric models to the observed radial profiles (e.g., Andrews et al. 2009; Isella et al. 2009). Spatially resolving the disks is important because it ensures that low millimeter spectral slopes are not just an artifact of high optical depth.

Today, mm spectral slopes are known for quite a number of disks, and spatially resolved observations indicate that the low values measured in these samples are related to grain growth (e.g., Testi et al. 2003; Natta et al. 2004; Rodmann et al. 2006). Grains are believed to collide and stick together by van der Waals forces, thus forming larger and larger aggregates (Dominik \& Tielens 1997; Poppe et al. 2000; Blum \& Wurm 2008). Due to this loose binding, collisions with velocities in excess of a few $\mathrm{m} \mathrm{s}^{-1}$ may lead to fragmentation of the aggregates.

Larger samples of radially resolved $\mathrm{mm}$ spectral slopes are expected in the near future, but so far no study interpreted $\mathrm{mm}$ observations using simulated grain size distributions. Instead simple parametric power-law distributions were used with an upper size cut-off. In this work, we use a state of the art dust grain evolution code (similar to Brauer et al. 2008a; Birnstiel et al. 2010) to derive steady-state grain distributions where grain growth and fragmentation effects balance each other. We selfconsistently solve for the grain size distributions and the disk structure to predict fluxes at $\mathrm{mm}$ wavelengths and the radial dependence of the mm spectral index. Comparing these results with observed values in the Taurus and Ophiuchus star-forming regions allows us to test the predictions of the theory of grain growth/fragmentation and to infer constraints on grain properties such as the critical collision velocity and the distribution of fragments produced in collision events.

Grains orbiting at the Keplerian velocity in a laminar gas disk feel a constant head wind (caused by the gas rotating slightly sub-keplerian), which forces them to spiral inwards 
(Weidenschilling 1977). If this drag is as efficient as the laminar theory predicts, all dust particles that are necessary to explain the observed spectral indices would quickly be removed (see Brauer et al. 2007). We therefore assume that the radial drift is halted by an unknown mechanism. Under this assumption, we find that the low values of the mm spectral index can be explained by the theory. We show that in order to explain the observed flux levels, the amount of observable ${ }^{1}$ dust needs to be reduced by either reducing the dust-to-gas ratio (perhaps by radial drift at a intermediate efficiency or during an earlier evolutionary epoch) or by dust particle growth beyond centimeter sizes.

\section{Model description}

\subsection{Disk model}

We consider disks around a PMS star with a mass of $0.5 M_{\odot}$, bolometric luminosity of $0.9 L_{\odot}$ and effective temperature of $4000 \mathrm{~K}$, at a distance of $140 \mathrm{pc}$, which are typical values for the sample of low-mass PMS stars studied in Ricci et al. (2010, hereafter R10). To derive the disk structure we adopted a modified version of the two-layer models of passively irradiated flared disks developed by Dullemond et al. (2001) (following the schematization by Chiang \& Goldreich 1997), in which we have relaxed the common assumption that dust grain properties are constant throughout the disk. For the disk surface density we adopted the self-similar solution for a viscous disk (see Lynden-Bell \& Pringle 1974) with parameters lying in the ranges observationally constrained by Andrews et al. (2009). The surface density gradient $\gamma$ and the characteristic radius $R_{\mathrm{c}}$ (for the definitions, see Hartmann et al. 1998) are assumed to be $\gamma=1$ and $R_{\mathrm{c}}=60 \mathrm{AU}$, respectively. Throughout this work we assume a constant dust-to-gas mass ratio of $1 \%$.

\subsection{Dust model}

We use a coagulation/fragmentation code as described in Brauer et al. (2008a) and Birnstiel et al. (2010) to simulate the growth of dust particles. Particles grow through mutual collisions (induced by Brownian motion and by turbulence, see Ormel \& Cuzzi 2007) and subsequent sticking by van der Waals forces. We assume the dust particles to be spheres of internal density $\rho_{\mathrm{S}}$ and vary $\rho_{\mathrm{s}}$ to account for porosity effects. However, we do not employ a dynamic porosity model (see Ormel et al. 2007; Zsom \& Dullemond 2008).

With increasing collision velocity $\Delta u$, the probability of sticking decreases and fragmentation events start to become important. Here, we use the same recipe for the fragmentation probability as in Birnstiel et al. (2010): particles above a fragmentation probability $u_{\mathrm{f}}$ are assumed to fragment while particles below $0.8 u_{\mathrm{f}}$ are assumed to stick. In between, we linearly interpolate between sticking and fragmentation. Recent studies of collision experiments (Güttler et al. 2010) and numerical simulations (Zsom et al. 2010) indicate that there is also a regime in which particles may bounce. However, because this topic is still not well understood, we will omit these effects here.

Radial drift is an as yet unsolved problem (Brauer et al. 2007; Birnstiel et al. 2009). Still there are several effects such as spiral wave structure (e.g., Cossins et al. 2009), density sinks

\footnotetext{
1 by "visible" or "observable" dust we mean the dust particles which are responsible for most of the thermal continuum emission at (sub-)mm wavelengths, which are typically smaller than a few centimeter in radius.
}

Table 1. Parameters of the model grid.

\begin{tabular}{lccccccc}
\hline \hline Parameter & \multicolumn{6}{c}{ Values } \\
\hline$M_{\text {disk }}$ & {$\left[M_{\odot}\right]$} & $5 \times 10^{-3}$ & $\mathbf{1} \times \mathbf{1 0}^{-\mathbf{2}}$ & $5 \times 10^{-2}$ & $1 \times 10^{-1}$ \\
$\alpha_{\mathrm{t}}$ & & $\mathbf{5} \times \mathbf{1 0}^{-\mathbf{4}}$ & $1 \times 10^{-3}$ & $5 \times 10^{-3}$ & - \\
$u_{\mathrm{f}}$ & {$[\mathrm{m} / \mathrm{s}]$} & 1 & $\mathbf{3}$ & 10 & - \\
$f_{\text {vac }}$ & {$[\%$ vol. $]$} & $\mathbf{1 0}$ & 30 & 50 & - \\
$\xi$ & & 1.0 & $\mathbf{1 . 5}$ & 1.8 & - \\
\hline
\end{tabular}

Notes. $M_{\text {disk }}$ is the total disk mass, $\alpha_{\mathrm{t}}$ is the turbulence parameter, $u_{\mathrm{f}}$ is the critical collision velocity, $f_{\mathrm{vac}}$ is the grain volume fraction of vacuum, and $\xi$ is the index of the distribution of fragments. The parameters of the fiducial model are highlighted in bold face.

(e.g., Brauer et al. 2008b), or zonal flows (e.g., Johansen et al. 2009) which may reduce the effectiveness of radial drift. We assume that radial drift is ineffective because we focus on the question whether observations can be explained through the physics of grain growth and fragmentation. The question to answer in this case is not how to retain particles at these radii, but rather how to create them there in the first place.

To investigate this problem, we simulate the physics of particle growth and fragmentation until a steady state between both processes develops. Because the relative velocities for particles typically increase with grain radius, we can relate the fragmentation velocity to a certain grain size (which defines the "fragmentation barrier", see Birnstiel et al. 2009)

$a_{\max } \simeq \frac{2 \Sigma_{\mathrm{g}}}{\pi \alpha_{\mathrm{t}} \rho_{\mathrm{s}}} \cdot \frac{u_{\mathrm{f}}^{2}}{c_{\mathrm{s}}^{2}}$

above which particles fragment (with $\Sigma_{\mathrm{g}}, \alpha_{\mathrm{t}}$ and $c_{\mathrm{s}}$ being the gas surface density, the turbulence parameter, and the sound speed, respectively). $u_{\mathrm{f}}$ and $\alpha_{\mathrm{t}}$ are assumed to be radially constant with $\alpha_{\mathrm{t}}$ values within a range expected from theoretical (see Johansen \& Klahr 2005; Dzyurkevich et al., 2010, in press) and observational works (see Andrews et al. 2009). Grains which reach $a_{\max }$ will experience high velocity collisions, causing them to be eroded or even completely fragmented. The resulting fragments can again contribute to growth processes at smaller sizes, and the grain size distribution will at some point reach a steady state where gain and loss terms caused by coagulation and by fragmentation cancel out at all sizes.

Particles will need a certain time to grow to the fragmentation barrier. The time to reach the steady state will therefore be several of these growth time scales. Depending on the distance to the central star, the steady state is typically reached after a few thousand years at $1 \mathrm{AU}$ up to about $1 \mathrm{Myr}$ at $100 \mathrm{AU}$. The mean ages of the sources in our sample are $\approx 2$ Myr and $\approx 0.5-1$ Myr for the Taurus and Ophiuchus PMS stars, respectively. Since radii around 40-80 AU dominate the observed emission at (sub-)mm wavelengths, we expect most of the samples to be in or at least close to a steady state.

If the highest collision velocity that turbulent motion induces (depending on $\alpha_{\mathrm{t}}$ and $c_{\mathrm{s}}$ ) is lower than the critical collision velocity $u_{\mathrm{f}}$, then (at least some) particles do not fragment (i.e. the break through the fragmentation barrier) and a steady state is never reached. Owing to this scenario, some of the possible combinations of the parameter values (see Table 1) do not reach a steady state and are therefore not included in the results.

The shape of the steady-state grain size distributions is influenced mainly by five parameters: the previously mentioned $\alpha_{\mathrm{t}}$, $u_{\mathrm{f}}, \Sigma_{\mathrm{g}}$, the temperature $T$ (through the sound speed $c_{\mathrm{s}}$ ), and by the prescription of fragmentation. In our models, we assume the distribution of fragments to follow a power-law number density 
distribution, $n(m) \propto m^{-\xi}$, with an upper end at $m_{\mathrm{f}}$. We consider fragmentation and cratering, as described in Birnstiel et al. (2010). Recent experiments suggest $\xi$ values between 1.07 and 1.37 (see Güttler et al. 2010). In this work, we consider $\xi$ values between 1.0 and 1.8 .

To calculate the dust opacity of a given grain size distribution we adopted the same dust grain model as in R10, i.e. porous composite spherical grains made of astronomical silicates, carbonaceous materials and water ices (see R10 for the references to the optical constants). The ratio between the fractional abundance of each species comes from Semenov et al. (2003), and models with three different porosities have been considered in this Letter (see Table 1). We used the Bruggeman mixing theory to combine the refractive indices of the different materials and to calculate the dust opacity of the composite grains. The opacity induces probably the largest uncertainties in our calculations because grain composition, grain structure and temperature effects may lead to largely different opacities (see, for example Henning \& Stognienko 1996).

\subsection{Comparison to observations}

We compare the (sub-)mm SED generated by our models with observational data of R10 and Ricci et al. (in prep.), more specifically the flux at $1 \mathrm{~mm}\left(F_{1 \mathrm{~mm}}\right)$ and the spectral index between 1 and $3 \mathrm{~mm}\left(F(\lambda) \propto \lambda^{-\alpha_{1-3} \mathrm{~mm}}\right)$. The samples considered include all class II disks in the Taurus-Auriga and $\rho$-Oph starforming regions for which both the central PMS star and the disk are observationally well characterized through optical-NIR spectroscopy/photometry and (sub-)mm photometry/interferometry. To calculate the dust opacity as a function of wavelengths and radius, and the temperature in the disk mid-plane, we iterated the two-layer disk model (keeping the profile of $\Sigma_{\mathrm{g}}$ constant in time) with the dust model described above until convergence is reached. Once the physical structure of the disk is determined, the two-layer disk models return the disk SED, which can be compared with the observations.

The influence of the different parameters on the calculated $\alpha_{1-3 \mathrm{~mm}}$ values can mostly be understood by a simple model for a dust distribution, as used in R10 (cf. Fig. 3 in R10): for maximum grain sizes much smaller than the observed wavelengths, the spectral index of the dust opacity $\beta_{1-3 \mathrm{~mm}}\left(\kappa(\lambda) \propto \lambda^{-\beta_{1-3} \mathrm{~mm}}\right)$ is constant, while it decreases for maximum particle sizes larger than sub-mm. In between (at a few tenth of a mm), there is a peak which is caused by an increased opacity of grains with sizes similar to the observed wavelength. The relation between $\alpha_{1-3 \mathrm{~mm}}$ and $\beta_{1-3 \mathrm{~mm}}$ depends on the emitting spectrum and the optical depth. For a completely optically thin disk in the Rayleigh-Jeans regime $\beta_{1-3} \mathrm{~mm}=\alpha_{1-3 \mathrm{~mm}}-2$. However, if the emitted spectrum deviates from the Rayleigh-Jeans limit, then $\beta_{1-3 \mathrm{~mm}} \gtrsim \alpha_{1-3 \mathrm{~mm}}-2$. In our models, $\alpha_{1-3 \mathrm{~mm}}-\beta_{1-3 \mathrm{~mm}}$ turns out to be typically between 1.4 and 1.7 if $a_{\max }$ is outside of the peak of opacity.

\section{Results}

\subsection{Sub-mm fluxes and spectral indices}

For all combinations of the parameters shown in Table 1, we solved for the steady-state grain size distributions and derived $\alpha_{1-3 \mathrm{~mm}}$ and $F_{1 \mathrm{~mm}}$. As noted before, some of the models do not result in a steady state and are therefore not shown here.

The top left panel of Fig. 1 shows the influence of $\alpha_{\mathrm{t}}$. According to Eq. (1), the maximum grain size increases if $\alpha_{\mathrm{t}}$

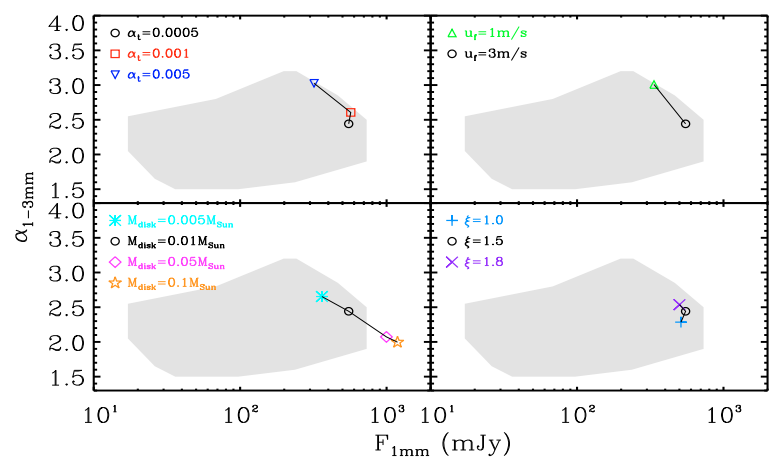

Fig. 1. Influence of the parameters $\alpha_{\mathrm{t}}$ (top left), fragmentation velocity (top right), disk mass (bottom left) and grain porosity (bottom right) on the observed fluxes and spectral indices. The black circle denotes the fiducial model whose parameters are given in Table 1 . The grey area represents the region in which the observed sources lie (see Fig. 2).

decreases. Depending on where $a_{\max }$ lies with respect to the opacity peak (see R10, Fig. 3), $\alpha_{1-3} \mathrm{~mm}$ can increase or decrease with increasing $\alpha_{\mathrm{t}}$. In the simulations presented here, $a_{\max }$ is typically so large that increasing $\alpha_{\mathrm{t}}$ predicts steeper spectral slopes.

$a_{\max }$ is more sensitive to $u_{\mathrm{f}}$ (cf. top right panel in Fig. 1): the maximum grain size $a_{\max }$ is proportional to $u_{\mathrm{f}}^{2}$, therefore a change of $u_{\mathrm{f}}$ by a factor of about 3 significantly changes $\alpha_{1-3} \mathrm{~mm}$ by increasing the grain size by about one order of magnitude. However many models with a fragmentation velocity of $10 \mathrm{~m} / \mathrm{s}$ never reach a steady state. It is therefore not possible to explain lower $\alpha_{1-3} \mathrm{~mm}$ values by a further increase of $u_{\mathrm{f}}$ alone.

The influence of $M_{\text {disk }}$ on $F_{1 \mathrm{~mm}}$ and $\alpha_{1-3 \mathrm{~mm}}$ is twofold. Firstly, a decrease in $M_{\text {disk }}$ (assuming a constant dust-to-gas ratio and a fixed shape of the disk surface density, i.e. not varying $R_{\mathrm{c}}$ and $\gamma$, see Sect. 2.1) reduces the amount of emitting dust and thus $F_{1 \mathrm{~mm}}$. Secondly, such a reduction in gas mass also reduces $a_{\max }$ (Eq. (1)), which tends to increase $\alpha_{1-3 \mathrm{~mm}}$. This combined trend is seen in Fig. 1. Hence, in order to explain faint sources with low $\alpha_{1-3 \mathrm{~mm}}$, the amount of emitting dust has to be reduced while the disk gas mass stays large. This effect could be achieved in two ways: the amount of dust could be reduced by radial drift at a reduced rate (full radial drift would quickly remove all mmsized grains, see Brauer et al. 2007) or only the "visible" amount of dust is reduced if some of the dust is already contained in larger bodies. This latter case is predicted by our non-steadystate distribution models and will be discussed in more detail in a forthcoming paper.

In general, lower values of $\xi$ translate to shallower grain-size distribution, which results in lower values of $\beta_{1-3 \mathrm{~mm}}$ (see Draine 2006). The lower right panel in Fig. 1 does not seem to indicate a strong dependence on $\xi$, but lower values of $\xi$ (around 1) seem to be closer to the observations especially at high fluxes.

Figure 2 shows the areas which are covered by our sets of simulations for different porosities in comparison to the observational samples. It can be seen that only the brightest sources are covered by the simulations. The trend of larger $\alpha_{1-3 \mathrm{~mm}}$ for a larger vacuum fraction seems to be in contradiction with Eq. (1), because smaller grain volume density leads to larger $a_{\max }$. However in this case, the opacity is much more affected by changing the grain structure: reducing the grains' vacuum fraction increases the spectral index at sub-mm, while it is reduced for longer wavelengths. Therefore opacity effects outweigh the smaller changes in $a_{\max }$. A more thorough analysis of opacity effects is beyond the scope of this letter, but it seems implausible that the large spread in the observations could be explained by different kinds of grains alone (see Draine 2006). 


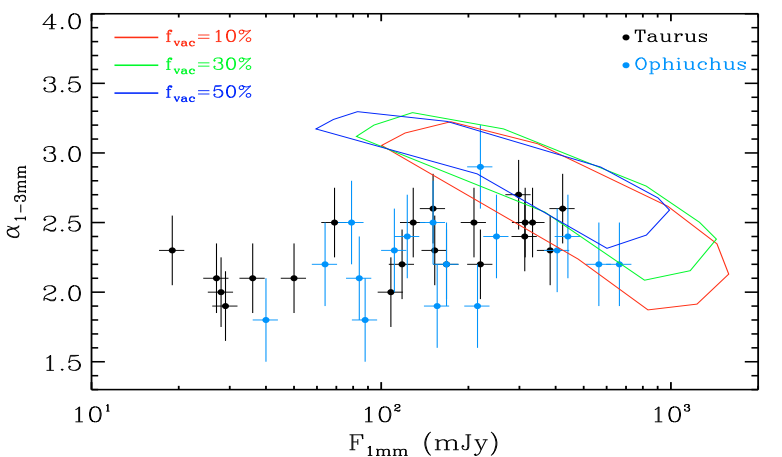

Fig. 2. Observed fluxes at mm-wavelengths of the Taurus (black dots) and the Ophiuchus (blue dots) star-forming regions (see R10 and Ricci et al. 2010, in prep.) and the areas covered by the simulation results for different vacuum fractions of the grains (varying all other parameters according to Table 1).

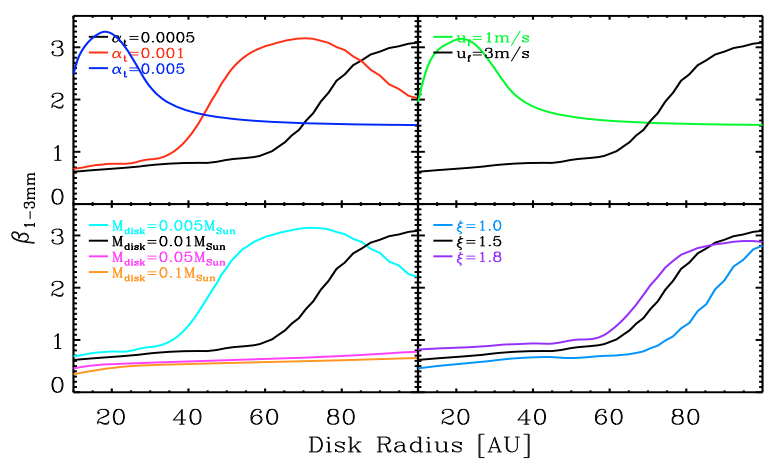

Fig. 3. Predicted profiles of the dust opacity index at mm-wavelengths for different variations of the fiducial model. The colors correspond to the parameters shown in Fig. 1.

\subsection{Radial profiles of the dust opacity index}

The presented models also compute $\alpha_{1-3} \mathrm{~mm}$ as function of radius. From the point of view of a comparison with the observations, this is somewhat premature because observational methods are not yet able to provide reliable radial profiles of $\alpha_{1-3 \mathrm{~mm}}$ (e.g., Isella et al. 2010; Banzatti et al., in prep.). Yet, the predicted radial dependence of $\beta_{1-3 \mathrm{~mm}}$ (shown in Fig. 3) agrees with the observations so far. It can be seen that the shape of most models looks similar, slightly increasing from $\beta_{1-3} \mathrm{~mm}$-values around 0.5 at $10 \mathrm{AU}$ up to around 1.5 at $100 \mathrm{AU}$. The reason for this is that $a_{\max }$ depends on the ratio of surface density over temperature. Under typical assumptions, $a_{\max }$ will decrease with radius. An upper grain size, which is decreasing with radius and stays outside the peak in the opacity, results in $\beta_{1-3} \mathrm{~mm}$ increasing with radius (cf. Fig. 3 in R10). If the radially decreasing upper grain size $a_{\max }$ reaches sizes just below $\mathrm{mm}$, then the peak in opacity will produce also a peak in the radial profile of $\beta_{1-3 \mathrm{~mm}}$ (the size of which depends much on the assumed opacity), which can be seen in Fig. 3. Thus, even though $a_{\max }$ is monotone in radius, $\beta_{1-3} \mathrm{~mm}$ does not need to be monotone.

\section{Discussion and conclusions}

We present the first comparison of simulated grain size distributions and observed mm spectral indices of YSOs in the Taurus and the Ophiuchus star-forming regions. Additionally we present the first predictions of the radial profile of the dust opacity index at $\mathrm{mm}$ wavelength that are consistent with the limits set by Isella et al. (2010).
Low values of the observed mm-slopes are quite naturally reproduced by our models, favoring low values of $\xi$ and $\alpha_{\mathrm{t}}$ as well as fragmentation threshold velocities above $1 \mathrm{~m} \mathrm{~s}^{-1}$. However, a simultaneous match to the observed range of flux levels requires a reduction of the dust mass by a factor of a few up to about 30. This over-prediction of fluxes cannot be fixed by simply reducing the disk mass because the predicted $\alpha_{1-3} \mathrm{~mm}$ would be too large for smaller disk masses. Opacities induce a large uncertainty in the flux levels. However, considering the results of Draine (2006), it seems implausible that the large spread in observed fluxes for different disks with similar $\alpha_{1-3} \mathrm{~mm}$ (which is probably even larger as very faint disks are not contained in the sample) can be explained by different grain mineralogy alone.

The aforementioned reduction of observable dust could be due to radial drift at a reduced rate or during an earlier epoch (drift has been artificially suppressed in this work in order to explain the low values of $\alpha_{1-3} \mathrm{~mm}$ by $\gtrsim 1 \mathrm{~mm}$ sized grains). Another possible explanation is grain growth to even larger sizes, as these bodies have a small opacity coefficient per unit mass.

Finally, a different dependence between $\alpha_{1-3} \mathrm{~mm}$ and the observed flux $F_{1 \mathrm{~mm}}$ might also originate from disk surface densities profiles that differ from what we have assumed in this work. This possibility, as well as the effect of a different dust composition, will be considered in a future work.

Acknowledgements. We thank Henrik Beuther and Jürgen Blum for useful discussions. L.R. and A.Z. acknowledge support of IMPRS. C.W.O. is supported by a grant from the Alexander von Humboldt foundation. We also thank Andrea Isella for a helpful referee report.

\section{References}

Andrews, S. M., Wilner, D. J., Hughes, A. M., Qi, C., \& Dullemond, C. P. 2009, ApJ, 700, 1502

Beckwith, S. V. W., Sargent, A. I., Chini, R. S., \& Guesten, R. 1990, AJ, 99, 924

Birnstiel, T., Dullemond, C. P., \& Brauer, F. 2009, A\&A, 503, L5

Birnstiel, T., Dullemond, C. P., \& Brauer, F. 2010, A\&A, 513, 79

Blum, J., \& Wurm, G. 2008, ARA\&A, 46, 21

Brauer, F., Dullemond, C. P., Johansen, A., et al. 2007, A\&A, 469, 1169

Brauer, F., Dullemond, C. P., \& Henning, T. 2008a, A\&A, 480, 859

Brauer, F., Henning, T., \& Dullemond, C. P. 2008b, A\&A, 487, L1

Chiang, E., \& Goldreich, P. 1997, ApJ, 490, 368

Cossins, P., Lodato, G., \& Clarke, C. J. 2009, MNRAS, 393, 1157

Dominik, C., \& Tielens, A. G. G. M. 1997, ApJ, 480, 647

Draine, B. T. 2006, ApJ, 636, 1114

Dullemond, C. P., Dominik, C., \& Natta, A. 2001, ApJ, 560, 957

Güttler, C., Blum, J., Zsom, A., Ormel, C. W., \& Dullemond, C. P. 2010, A\&A, 513,56

Hartmann, L., Calvet, N., Gullbring, E., \& D’Alessio, P. 1998, ApJ, 495, 385

Henning, T., \& Stognienko, R. 1996, A\&A, 311, 291

Isella, A., Carpenter, J. M., \& Sargent, A. I. 2009, ApJ, 701, 260

Isella, A., Carpenter, J. M., \& Sargent, A. I. 2010, ApJ, 714, 1746

Johansen, A., \& Klahr, H. 2005, ApJ, 634, 1353

Johansen, A., Youdin, A., \& Klahr, H. 2009, ApJ, 697, 1269

Lynden-Bell, D., \& Pringle, J. E. 1974, MNRAS, 168, 603

Natta, A., Testi, L., Neri, R., Shepherd, D. S., \& Wilner, D. J. 2004, A\&A, 416, 179

Ormel, C. W., \& Cuzzi, J. N. 2007, A\&A, 466, 413

Ormel, C. W., Spaans, M., \& Tielens, A. G. G. M. 2007, A\&A, 461, 215

Poppe, T., Blum, J., \& Henning, T. 2000, ApJ, 533, 454

Ricci, L., Testi, L., Natta, A., et al. 2010, A\&A, 512, 15

Rodmann, J., Henning, T., Chandler, C. J., Mundy, L. G., \& Wilner, D. J. 2006, A\&A, 446, 211

Semenov, D., Henning, T., Helling, C., Ilgner, M., \& Sedlmayr, E. 2003, A\&A, 410,611

Strom, K. M., Strom, S. E., Edwards, S., Cabrit, S., \& Skrutskie, M. F. 1989, AJ, 97, 1451

Testi, L., Natta, A., Shepherd, D. S., \& Wilner, D. J. 2003, A\&A, 403, 323

Weidenschilling, S. J. 1977, MNRAS, 180, 57

Zsom, A., \& Dullemond, C. P. 2008, A\&A, 489, 931

Zsom, A., Ormel, C. W., Güttler, C., Blum, J., \& Dullemond, C. P. 2010, A\&A, 513,57 